\title{
An Efficient Storage of Infrared Video of Drone Inspections via Iterative Aerial Map Construction
}

\author{
Belyaev, Evgeny; Forchhammer, Soren
}

Published in:

IEEE Signal Processing Letters

Link to article, DOI:

10.1109/LSP.2019.2921250

Publication date:

2019

Document Version

Peer reviewed version

Link back to DTU Orbit

Citation (APA):

Belyaev, E., \& Forchhammer, S. (2019). An Efficient Storage of Infrared Video of Drone Inspections via Iterative Aerial Map Construction. IEEE Signal Processing Letters, 26(8), 1157-1161. [8733128]. https://doi.org/10.1109/LSP.2019.2921250

\section{General rights}

Copyright and moral rights for the publications made accessible in the public portal are retained by the authors and/or other copyright owners and it is a condition of accessing publications that users recognise and abide by the legal requirements associated with these rights.

- Users may download and print one copy of any publication from the public portal for the purpose of private study or research.

- You may not further distribute the material or use it for any profit-making activity or commercial gain

- You may freely distribute the URL identifying the publication in the public portal 


\title{
An efficient storage of infrared video of drone inspections via iterative aerial map construction
}

\author{
Evgeny Belyaev and Søren Forchhammer
}

\begin{abstract}
In this letter we present a novel compression algorithm of infrared video sequences captured during drone inspections based on iterative aerial map construction. In our approach we first apply a stitching algorithm to construct a map of an inspected area assuming that a drone is flying at the same altitude by trajectory close to meander, so that each frame can have a partial overlap with other frame captured much earlier or later. Then we extract position and rotation angle within the map for each frame and use them as a side information for the video coding. In order to compress an input video sequence, we utilize a Multi-view H.265/HEVC with two views. First view is a virtual view generated utilizing the decoded frames of the second view and the side information, while the input video is considered as the second view, which is encoded utilizing the virtual view as a reference for the interview prediction. The proposed approach has two main benefits. First, the aerial map is generated during decoding utilizing the side information, i.e., the map is not embedded into a bit stream. Second, the inter-view prediction allows to exploit an additional redundancy which is typical for a drone video. Experimental results show that the proposed algorithm provides $1.4-2.4 \%$ bit rate savings comparing to $\mathrm{H.265} / \mathrm{HEVC}$. The maximum possible bit rate savings are estimated from 15.5 to $18.9 \%$ assuming that the drone is repeatedly flying many times at exactly the same trajectory.
\end{abstract}

Index Terms-infrared video, video coding, H.265/HEVC

\section{INTRODUCTION}

$\mathbf{I}$ $\mathrm{n}$ recent years, more and more local area inspections are performed utilizing unmanned airborne vehicles such as quadcopters, hexacopters or light fixed wing planes called drones. These drones can be efficiently used for leakages detection in district heating systems [1], damages detection of solar panels fields [2], in agricultural monitoring [3] and so on. For such inspections, an infrared camera is mounted on a drone and used for recording of video data. In order to cover the whole inspected area the drone is flying approximately at the same altitude by trajectory close to meander with partial frames overlapping, so that an aerial map can be built using stitching algorithms. The aerial map can be used for further macro analysis of the area, while the recorded video frames are needed for more detailed analysis of a particular location or object. In a basic scenario (scenario 1) we could store

Copyright (c) 2017 IEEE. Personal use of this material is permitted. However, permission to use this material for any other purposes must be obtained from the IEEE by sending a request to pubs-permissions@ieee.org

This research was supported in part by the Government of the Russian Federation through the ITMO Fellowship and Professorship Program, and in part by the Danish energy technological development and demonstration program (EUDP), EUDP 15-I, 64015-0072.

Evgeny Belyaev is with the International Laboratory "Computer Technologies”, ITMO University, Russia, e-mail: eabelyaev@itmo.ru

Søren Forchhammer is with the Department of Photonics Engineering, Technical University of Denmark, e-mail: sofo@fotonik.dtu.dk only the recorded video in an inspection data base. However, in this case we need to apply a high-complexity stitching algorithm each time, when the macro analysis is required. Therefore, it is preferable to store both the recorded video and the corresponding aerial map (scenario 2) in the data base.

Both a recorded drone video and the corresponding aerial map can be compressed separately via existing image and video coding algorithms, e.g., H.265/HEVC [4] video coding standard (in inter-mode for a video and in intra-mode for a map). However, it is not efficient from compression performance point of view, because the video and the aerial map are correlated and should be compressed jointly. Similarly to video coding using long-term background reference [5], [6], [7], [8], in [9] we introduced drone infrared video coding algorithm based on aerial map prediction (AMP). In this approach we first compress an aerial map as a still image using H.265/HEVC in intra-mode. Then we apply global motion estimation to extract the most similar frame from the reconstructed aerial map and use it as an extra reference frame in the list of reference frames of H.265/HEVC. It was shown that a motion compensation using the extended reference list can significantly reduce the drone video bit rate. In particular case of drone videos with camera rotation only, the joint bit rate of both the drone video and the aerial map is up to $35 \%$ lower than the bit rate of the drone video provided by conventional H.265/HEVC coding. It is possible, because a drone video has a camera rotation which cannot be well estimated by blockbased motion estimation, and a drone can fly many times above the same area, but the number of reference frames allowed by H.265/HEVC is not enough to use the similarity between current frame and frames captured few seconds or minutes ago. However, in general, the approach from [9] does not give compression benefits comparing to conventional H.265/HEVC coding, since the map bit rate may be higher than bit rate savings provided by AMP. For dashcam video coding, in [10] it was proposed to store a huge previously recorded video set covering wide area and long time (historical data) at both the encoder and decoder. The historical data is used to generate the most similar frame for each frame of the current video. Then the generated frames are considered as a base view of the 3D-HEVC encoder, while the current video is considered as a second view and encoded utilizing inter-view prediction. Similar approach was applied in [11], [12] for efficient Iframes coding for satellite video, where Google Earth is used as historical data. However, such approaches cannot be applied if the historical data for a given area is not collected.

In order to overcome these limitations, in this letter we propose to build the aerial map at the encoder and the decoder 
sides simultaneously using side information extracted from a stitching algorithm. Since the drone is flying approximately at the same altitude, this side information includes planar position and rotation angle for each frame within the aerial map which are embedded into a compressed bit stream. In order to compress an input video sequence, we utilize a Multiview H.265/HEVC (MV-HEVC) with two views. First view is a virtual view containing frames extracted from the current version of the aerial map constructed utilizing already decoded frames of the second view and the side information. The input video is considered as the second view and compressed using the virtual view as a reference for the inter-view prediction. As a result, the aerial map is not embedded into the compressed bit stream, since both encoder and decoder iteratively construct it during encoding (or decoding) utilizing the side information and reconstructed frames (scenario 3). This coding scheme does not require the MV-HEVC encoder/decoder modifications, i.e., it is compatible with the standard. Since the available stitching algorithms (for example, see [13]) do not provide the side information described above, we developed our own algorithm which is based on mask-based image blending as proposed in [14]. Experimental results on infrared video sequences captured during drone inspections show that the proposed algorithm provides $1.4-2.4 \%$ bit rate savings. Herewith, the maximum possible bit rate savings are estimated from 15.5 to $18.9 \%$ assuming that the drone is repeatedly flying many times at exactly the same trajectory.

The rest of the letter is organized as follows. Section II presents the proposed compression algorithm. Section III introduces the proposed stitching algorithm for infrared video sequences of drone inspections. Section IV provides rate-distortion comparison of the proposed approach with H.265/HEVC. Section V concludes the presented results.

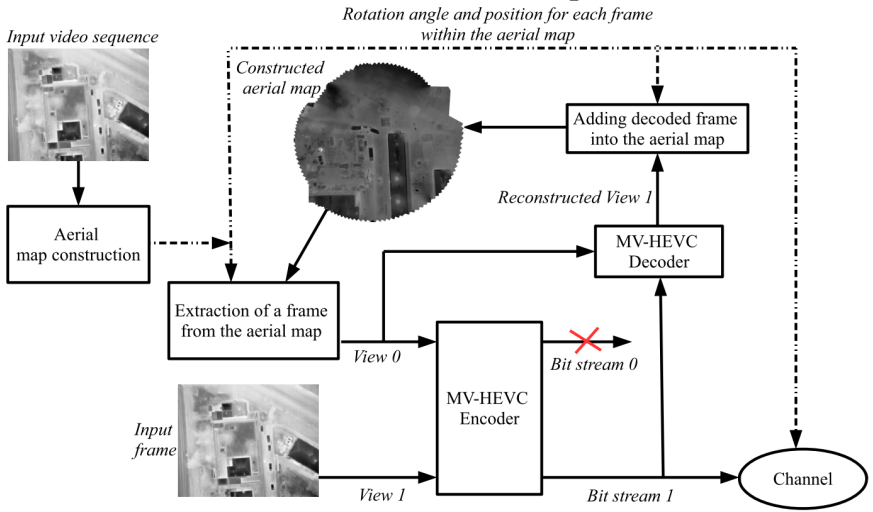

Fig. 1: Proposed encoding scheme

II. PRoposed CODING ALGORITHM

Figure 1 shows the proposed compression algorithm. We assume that each pixel $x$ of an input infrared frame is represented by an integer value within the range $0 \leq x \leq$ 255. First, an input infrared video sequence is used by a stitching algorithm to build an aerial map. The stitching is performed under assumption that a drone is flying at the same altitude by trajectory close to meander with partial frames overlapping, i.e., planar motion model is assumed. Let us sort all video frames in an encoding (decoding) processing order, so that frames with indices $f-1, f-2, \ldots$ are encoded (decoded) before frame with index $f$. For a frame with index $f$ the stitching algorithm provides position $\left\{x_{f}, y_{f}\right\}$ and rotation angle $\alpha_{f}$ which are embedded into a video bit stream. Then, encoding of the group of frames (GOP) with indices $f, f+1, \ldots, f+G$ is performed in the following way. First, the encoder extracts frames at positions $\left\{x_{f}, y_{f}\right\},\left\{x_{f+1}, y_{f+1}\right\}, \ldots,\left\{x_{f+G}, y_{f+G}\right\}$ and rotated at the corresponding angles $\alpha_{f}, \alpha_{f+1}, \ldots, \alpha_{f+G}$ from the map and forming $G$ frames of the virtual view. Then, the second view containing input frames $f, f+1, \ldots, f+G$ are encoded using the virtual view as a reference for the inter-view prediction. Finally, the reconstructed frames $f, f+1, \ldots, f+G$ and corresponding positions $\left\{x_{f}, y_{f}\right\},\left\{x_{f+1}, y_{f+1}\right\}, \ldots,\left\{x_{f+G}, y_{f+G}\right\}$ and angles $\alpha_{f}, \alpha_{f+1}, \ldots, \alpha_{f+G}$ are utilized to update the aerial map as it is defined by the stitching algorithm. At the decoder side, the aerial map is updated and the virtual view is generated in the same way.

The proposed coding scheme has the following features:

1) The decoder can reconstruct both video sequence and the corresponding aerial map, i.e., additional resources for the map storage are not needed.

2) An aerial map allows to take into account a camera rotation model, and enables motion-compensated prediction from frames captured a long time ago. This helps to exploit additional redundancy of video sequences captured during drone inspections, i.e., it improves the coding efficiency.

3) The coding performance of the proposed scheme depends on the flying trajectory. For example, if a drone is always flying without camera rotation and does not return back to the same area, then the proposed scheme will not provide significant benefits comparing to the conventional video coding schemes. And vice versa, the maximum improvements will be achieved if the drone is repeatedly flying exactly the same trajectory.

4) The coding performance of the proposed scheme also depends on a performance of the stitching algorithm.

\section{PROPOSED STITCHING ALGORITHM}

\section{A. Global motion estimation}

The proposed stitching algorithm utilizes global motion estimation (GME) between infrared frames and between a frame and an aerial map. In order to improve convergence and reduce computational complexity, similarly to [15], we use a low-pass image pyramid approach, when the motion parameters are estimated on down-scaled images and the estimated parameters are enhanced on up-scaled images. Taking into account the camera motion model introduced above, we can estimate rotation angle, horizontal and vertical displacements which minimize the sum of squared differences (SSD) between the frames. However, SSD-based motion estimation can select motion vectors ignoring low amplitude areas (which have relatively low weight in SSD metric). As a result, the SSD applied directly to infrared frames could provide incorrect motion parameters.

In order to improve robustness of GME, we propose to compute SSD on binary images in which both high and low amplitude areas have similar importance. Let us define 
$\mathbb{B}\left(F_{i}\right)$ as a binary image of frame $F_{i}$, where operator $\mathbb{B}($. is introduced in the following way. First, we apply a tone mapping operator. Let us define $x$ as a pixel value of an input infrared image and $\mathbb{T}(x)$ as a corresponding pixel in a tone mapped image. We divide the input image into overlapping blocks of size $N \times N$, so that pixel $x$ belongs to blocks $X_{1}, X_{2}, \ldots, X_{K}$. For each block $X_{i} \in\left\{X_{1}, X_{2}, \ldots, X_{K}\right\}$ we compute an amplifying coefficient as

$$
a_{i}=\frac{255}{\max \left(\frac{X_{i}-\min \left(X_{i}\right)}{\max \left(X_{i}\right)-\min \left(X_{i}\right)}\right)},
$$

and compute the tone mapped value as $\mathbb{T}(x)=\frac{x}{K} \sum_{i=1}^{K} a_{i}$. Then the corresponding pixel in the binary image is calculated as

$$
\mathbb{B}(x)=\left\{\begin{array}{l}
255, \text { if } \mathbb{T}(x)>T, \\
0, \text { otherwise, }
\end{array}\right.
$$

where $T$ is global image threshold obtained using Otsu's method (function graythresh in MATLAB). Figure 2 shows an example of a frame and corresponding binary image.

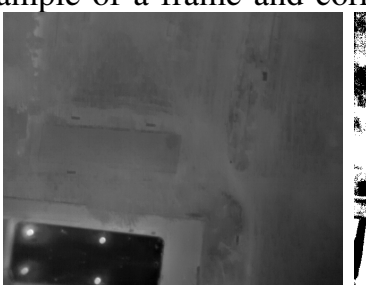

(a) $F_{i}$

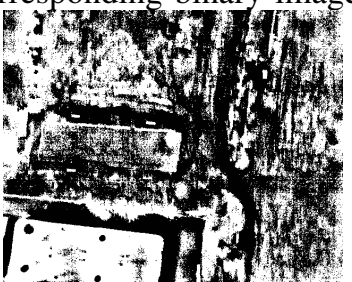

(b) $\mathbb{B}\left(F_{i}\right)$
Fig. 2: Infrared frame and corresponding binary image

\section{B. Aerial map construction}

The proposed aerial map construction is shown in Algorithm 1. This algorithm requires a list of frames indices in a processing order $\mathbf{p}=\left\{p_{1}, \ldots, p_{M}\right\}$ and corresponding list of reference frames indices $\mathbf{r}=\left\{r_{1}, \ldots, r_{M}\right\}$, where $M$ is a number of frames in a infrared video sequence. First, we apply GME between frames $F_{i}$ and $F_{j}$, where frame $F_{j}$ is a reference frame (which is already processed), and obtain relative displacements $\Delta x, \Delta y$ and rotation angle $\Delta \alpha$ (line 7). Then, we apply GME between frame $F_{i}$ and aerial map $A$ utilizing $\hat{x}_{i}=x_{j}+\Delta x, \hat{y}_{i}=y_{j}+\Delta y, \hat{\alpha}_{i}=\alpha_{j}+\Delta \alpha$ as an initial estimates (line 9). As a result, rotation angle $\alpha_{i}$ and coordinates $x_{i}, y_{i}$ for frame $F_{i}$ within map $A$ are determined (coordinates of the first frame are predetermined by values $x_{0}, y_{0}$ and $\alpha_{0}$ ). Finally, we update map $A$ utilizing mask-based image blending from [14] (lines 11-13). In this approach, each pixel in the map $A$ is computed as the weighted sum of all corresponding pixels having the same coordinates in the map. The weight matrix $W$ contains a weight of each pixel depending on its position within a frame: pixels which are closer to a frame center have higher weights. Here, operator $\mathbb{M}(F, x, y, \alpha)$ creates zero matrix of the same size as $A$, rotates frame $F$ by angle $\alpha$ and inserts it with coordinates $x, y$ into the created matrix. In line 13, the division is performed in an element by element way, and $\delta$ is a small value preventing division by zero. The stitching performance of Algorithm 1 depends on the order $\mathbf{p}$ of processing frames. In a simple case, $\mathbf{p}$ could describe the frame playback order, i.e., $\mathbf{p}=\{1,2, \ldots, M\}$, with $\mathbf{r}$ containing a list of corresponding previous frames

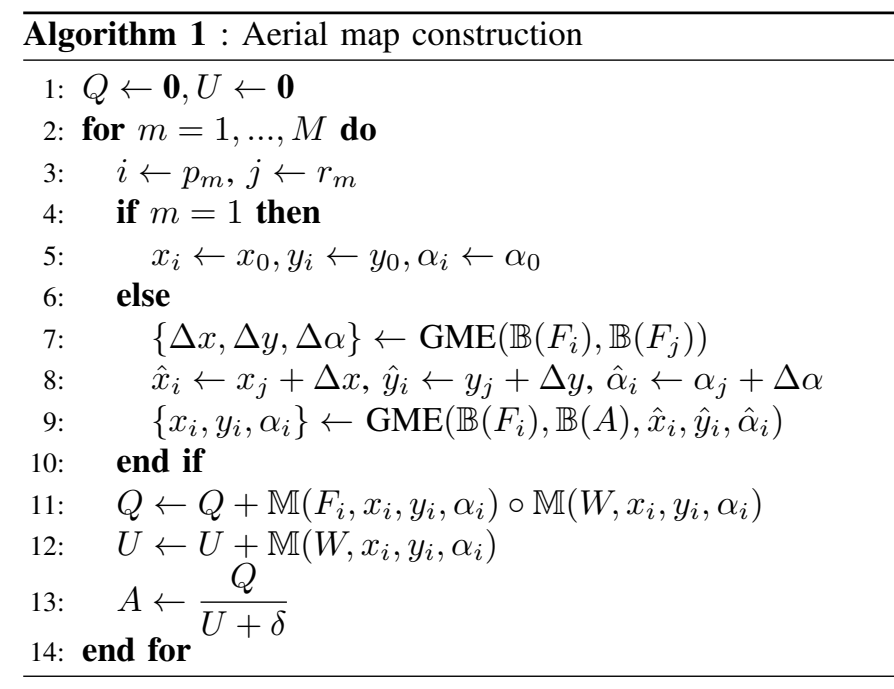

$\mathbf{r}=\{\varnothing, 1,2, \ldots, M-1\}$. However, our experiments show that in such a case GME in line 9 ignores frames which are close to $F_{i}$ in terms of distance, but are far in terms of playback order. As a result, an error in frame alignment can be accumulated, especially if hundreds of frames are involved. Therefore, in this letter we propose to proceed with the frames in a minimum distance order. In this approach, at the first iteration we are starting from a frame $F_{s}$ and searching for a frame $F_{k}$ which is playing back later than frame $F_{s+\Delta f}$ and has the minimum distance to frame $F_{s}$ according to GPS coordinates. If distortion $\operatorname{SSD}\left(\mathbb{B}\left(F_{s}\right), \mathbb{B}\left(F_{k}\right)\right)$ returned by GME obtained for frames $F_{s}$ and $F_{k}$ is lower than threshold $d_{\max }$, then we add frame $F_{k}$ into the list of frames processing order $\mathbf{p}$, and frame $F_{s}$ into the list of reference frames $\mathbf{r}$. Then we are searching for a frame $F_{m}$ which is playing back earlier than frame $F_{s-\Delta f}$ in the same way. If both of the frames do not provide SSD lower than $d_{\max }$, then we add frames $F_{s+1}$ and $F_{s-1}$ into the list $\mathbf{p}$, and frame $F_{s}$ into the list of reference frames $\mathbf{r}$. At the next iteration, we repeat all the procedures for each frame in the list $\mathbf{p}$. If after iteration $n$ the list $\mathbf{p}$ contains $M$ frames, then the algorithm is stopped. The starting frame index $s$ is selected to provide the minimum number of iterations $n$.

\section{Performance eValuation}

Experimental results were obtained for three test video sequences captured by Drone Systems ApS [16] during district heating inspection in the Copenhagen area usig a Flir Tau2 infrared camera with frame resolution $640 \times 512$, frame rate $9 \mathrm{~Hz}$. The test video sequences contain 412, 448 and 552 frames, respectively. Figures 3-4 shows corresponding aerial maps constructed by Algorithm 1 with parameters $N=32, \Delta f=18$ for all test sequences, and $d_{\max }$ equal to 18000,16500 and 15000 for test sequences $1,2,3$, respectively. GME was performed with minimum $10 \%$ overlap of frames. One can see that in some cases the maps have discontinuous areas marked by black color. The discontinuous appear, since frames which are close in terms of distance, but are far in terms of playback order, have an overlapping level significantly less than $10 \%$ which is too small for the proposed GME. 
TABLE I: Coding performance comparison

\begin{tabular}{|c|c|c|c|c|c|c|c|c|c|c|c|c|c|}
\hline & & \multicolumn{4}{|c|}{ Video 1} & \multicolumn{4}{|c|}{ Video 2} & \multicolumn{4}{|c|}{ Video 3} \\
\hline Coding scheme & $\mathrm{QP}$ & 25 & 30 & 35 & 40 & 25 & 30 & 35 & 40 & 25 & 30 & 35 & 40 \\
\hline HEVC (map only) & Bit rate, kbps & 94.8 & 58.3 & 36.0 & 22.2 & 96.4 & 59.1 & 36.3 & 22.3 & 85.8 & 52.8 & 32.6 & 20.0 \\
\hline \multirow{3}{*}{$H E V C$} & Bit rate, kbps & 362.9 & 170.6 & 89.8 & 50.2 & 371.2 & 173.4 & 91.8 & 50.5 & 368.4 & 171.1 & 90.3 & 50.1 \\
\hline & PSNR, dB & 36.45 & 34.60 & 32.48 & 30.23 & 36.45 & 34.58 & 32.43 & 30.12 & 36.36 & 34.50 & 32.40 & 30.16 \\
\hline & SSIM & 0.8879 & 0.8612 & 0.8295 & 0.7948 & 0.8882 & 0.8620 & 0.8304 & 0.7946 & 0.8867 & 0.8590 & 0.8265 & 0.7908 \\
\hline \multirow{4}{*}{ Proposed 1} & Bit rate, kbps & 358.4 & 167.1 & 87.8 & 48.8 & 368.9 & 171.3 & 90.5 & 49.8 & 365.4 & 169.0 & 88.1 & 48.7 \\
\hline & PSNR, dB & 36.44 & 34.59 & 32.46 & 30.21 & 36.45 & 34.56 & 32.41 & 30.10 & 36.36 & 34.50 & 32.38 & 30.13 \\
\hline & SSIM & 0.8878 & 0.8612 & 0.8295 & 0.7952 & 0.8882 & 0.8619 & 0.8302 & 0.7945 & 0.8867 & 0.8589 & 0.8264 & 0.7908 \\
\hline & BD-Rate [18], \% & \multicolumn{4}{|c|}{2.4} & \multicolumn{4}{|c|}{1.4} & \multicolumn{4}{|c|}{2.1} \\
\hline \multirow{4}{*}{ Proposed 2} & Bit rate, kbps & 318.4 & 140.3 & 69.3 & 36.4 & 333.3 & 148.2 & 74.6 & 39.3 & 328.1 & 144.4 & 71.8 & 38.1 \\
\hline & PSNR, dB & 36.41 & 34.53 & 32.37 & 30.11 & 36.42 & 34.51 & 32.33 & 30.02 & 36.32 & 34.43 & 32.30 & 30.05 \\
\hline & SSIM & 0.8874 & 0.8607 & 0.8295 & 0.7966 & 0.8878 & 0.8614 & 0.8300 & 0.7953 & 0.8862 & 0.8583 & 0.8262 & 0.7915 \\
\hline & BD-Rate [18], \% & \multicolumn{4}{|c|}{18.9} & \multicolumn{4}{|c|}{15.5} & \multicolumn{4}{|c|}{16.6} \\
\hline
\end{tabular}

TABLE II: Complexity and data storage capacity for three data base processing scenarios

\begin{tabular}{|c|c|c|c|c|c|}
\hline \multirow{2}{*}{ Scenario } & \multicolumn{2}{|l|}{ Data base preparation } & \multicolumn{2}{|l|}{ Data base utilization by a user } & \multirow{2}{*}{ Data storage capacity } \\
\hline & Processing & Complexity & Processing & Complexity & \\
\hline 1 & Video encoding & low & Video decoding and aerial map construction & high & $100 \%$ \\
\hline 2 & $\begin{array}{l}\text { Aerial map construction and encoding, } \\
\text { video encoding }\end{array}$ & high & Aerial map decoding, video decoding & low & $123-144 \%$ \\
\hline 3 & $\begin{array}{l}\text { Side information computation, video en- } \\
\text { coding utilizing the side information }\end{array}$ & high & $\begin{array}{l}\text { Video decoding with aerial map construc- } \\
\text { tion utilizing the side information }\end{array}$ & low & $\begin{array}{l}\text { 98-99\% (Proposed 1), } \\
81-85 \% \text { (Proposed 2). }\end{array}$ \\
\hline
\end{tabular}

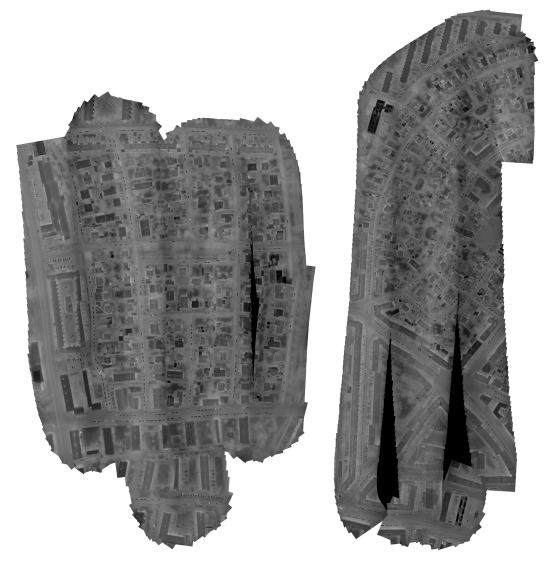

$\begin{array}{ll}\text { (a) Aerial map } 1 & \text { (b) Aerial map } 2\end{array}$

Fig. 3: Aerial maps obtained for Video 1 and Video 2

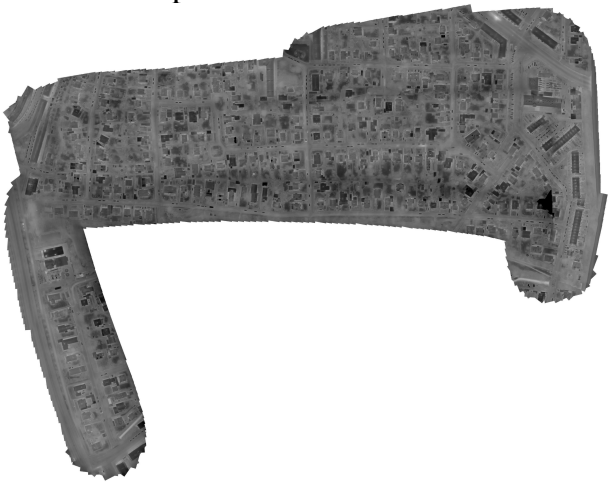

Fig. 4: Aerial map obtained for Video 3

Table I shows rate-distortion performance for different coding schemes implemented utilizing HTM-16.3 [17] which is a reference software of the Multi-view extension of H.265/HEVC [4]. The software was used with GOP size 8. Each GOP was encoded separately. Herewith, to avoid intraframe coding, the last reconstructed frame of each GOP was used as a first frame of the next GOP for both views. Here, HEVC (map only) shows the bit rate corresponding to the intracoding of an aerial map only (for reference), $H E V C$ means that each frame in the virtual view (except the first one in each GOP) is zero-frame. Proposed 1 means that each frame in the virtual view (except the first one in each GOP) is generated as it is described in Section II. The side information for each video was represented as a text file and compressed by 7-zip software [19], so that the resulting bit rate was approximately $0.6 \mathrm{kbps}$. One can see that Proposed 1 provides from 1.4 to $2.4 \%$ bit rate savings comparing to HEVC. Proposed 2 corresponds to the coding performance, when the drone is flying exactly the same trajectory many times, i.e., when the proposed algorithm should provide the maximum possible bit rate savings. To simulate it, we repeated each video sequence ones again, compressed it via Proposed 1 and measured the rate-distortion performance only for the second half of the resulting video sequence. In this case we can reach from 15.5 to $18.9 \%$ bit rate savings. Since Algorithm 1 utilizes image blending, the resulting aerial map is blurry (has less high frequency information than the original frames). Therefore, the inter-view prediction is less efficient at high bit rates.

Table II shows comparison of different data base organization scenarios in terms of complexity and required data storage capacity. At the user side, an aerial map construction for the proposed scenario 3 requires execution of lines 11-13 of Algorithm 1, i.e., rotation of frame $F_{i}$ and weight matrix $W$, and two additions, one multiplication and one division per pixel. It is less than in scenario 1, which needs to run a full stitching algorithm in order to build an aerial map. At the same time, the proposed scenario 3 requires less data storage capacity compared to scenarios 1 and 2 .

\section{CONCLUSION}

In this letter we presented a novel promising algorithm for drone infrared video coding based on iterative aerial map construction. It allows to avoid storage of an aerial map which can be build by a decoder utilizing reconstructed images of the video sequence and a side information. Moreover, the proposed aerial map prediction provides improvements of the coding efficiency which in magnitude depends on the drone flying trajectory and the map stitching algorithm. 


\section{REFERENCES}

[1] O.Friman, P.Follo et al., "Methods for Large-Scale Monitoring of District Heating Systems Using Airborne Thermography", IEEE Transactions on Geoscience and Remote Sensing, Vol.52, Iss.8, 2014.

[2] SungWon Lee, Kwang Eun An et al.,"Detecting faulty solar panels based on thermal image processing," IEEE International Conference on Consumer Electronics (ICCE), 2018.

[3] Jose A. J. Berni. et al.,"Thermal and Narrowband Multispectral Remote Sensing for Vegetation Monitoring From an Unmanned Aerial Vehicle," IEEE Transactions on Geoscience and Remote Sensing, vol.47, no.3, pp.722-738, 2009.

[4] High Efficiency Video Coding, document ITU-T Rec. H.265 and ISO/IEC 23008-2, 2014.

[5] A. Smolic et al, "Long-term global motion estimation and its application for sprite coding, content description, and segmentation", IEEE Transactions on Circuits and Systems for Video Technology, Vol.9, Issu.8, pp.1227-1242, 1999.

[6] X. Zhang, T. Huang, Y. Tian, and W. Gao, "Background-modeling-based adaptive prediction for surveillance video coding," IEEE Transactions on Image Processing, Vol. 23, No. 2, pp. 769-784, 2014.

[7] F. Chen, H. Li, L. Li, D. Liu, and F. Wu, "Block-composed background reference for high efficiency video coding," IEEE Transactions on Circuits and Systems for Video Technolgy, vol. 27, no. 12, pp. 2639-2651, 2017.

[8] G. Wang, B. Li, Y. Zhang, and J. Yang, "Background modeling and referencing for moving cameras-captured surveillance video coding in HEVC,' IEEE Transactions on Multimedia, Vol.27, Iss.12, pp.2639-2651, 2018.

[9] E.Belyaev and S.Forchhammer, "Drone HDR Infrared Video Coding via Aerial Map Prediction", 2018 IEEE International Conference on Image Processing., 2018.

[10] Ma Biao and A. Reibman, "DashCam Video Compression using Historical Data", Picture Coding Symposium (PCS), 2016.

[11] Xu Wang, Jing Xiao et al.,"Cruise UAV Video Compression Based on Long-Term Wide-Range Background", Data Compression Conference (DCC), page 466, 2017.

[12] X.Wang, R.Hu, Z.Wang, J.Xiao, Virtual Background Reference Frame Based Satellite Video Coding, IEEE Signal Processing Letters, Vol.25, Iss.10, 2018.

[13] Image Composite Editor [Online], https://www.microsoft.com/ en-us/research/product/computational-photography-applications/ image-composite-editor/

[14] Y. Xiong, K. Pulli, "Mask-based image blending and its applications on mobile devices", MIPPR 2009: Remote Sensing and GIS Data Processing and Other Applications, 2009.

[15] F.Dufaux, J.Konrad, Efficient, robust, and fast global motion estimation for video coding, IEEE Transactions on Image Processing, Vol.9, Iss.3, pp.497-501, 2000.

[16] Drone Systems ApS, http://dronesystems.dk/

[17] MV-HEVC Software [Online], https://hevc.hhi.fraunhofer.de/3dhevc/

[18] G. Bjontegaard, "Calculation of average PSNR differences between RDcurves", VCEG-M33, Thirteenth Meeting of the Video Coding Experts Group (VCEG): Austin, Texas, USA, 2-4 April, 2001.

[19] 7-Zip file archiver [Online],https://www.7-zip.org/ 